\title{
Circuit
}

Musiques contemporaines

\section{Introduction. Bienvenue dans le continuum de l'improvisation}

\section{Clément Canonne et Maxime McKinley}

Volume 30, numéro 2, 2020

Le continuum de l'improvisation

URI : https://id.erudit.org/iderudit/1071117ar

DOI : https://doi.org/10.7202/1071117ar

Aller au sommaire du numéro

Éditeur(s)

Circuit, musiques contemporaines

ISSN

1183-1693 (imprimé)

1488-9692 (numérique)

Découvrir la revue

Citer ce document

Canonne, C. \& McKinley, M. (2020). Introduction. Bienvenue dans le continuum de l'improvisation. Circuit, 30(2), 5-9. https://doi.org/10.7202/1071117ar d'utilisation que vous pouvez consulter en ligne.

https://apropos.erudit.org/fr/usagers/politique-dutilisation/ 


\section{Introduction. Bienvenue dans le continuum de l'improvisation}

Clément Canonne et Maxime McKinley

Dans la collection de Circuit, qui célèbre cette année son $30^{\mathrm{e}}$ anniversaire, trois numéros en particulier sont liés à ce qu'il n'est pas exagéré d'appeler de vives polémiques, parmi lesquelles deux concernent directement les musiques improvisées et l'émergence montréalaise de la musique dite «actuelle» (notons que si, en France, ce terme renvoie généralement aux musiques populaires amplifiées, au Québec il désigne plutôt les scènes underground et l'improvisation). Nos lecteurs assidus savent sans doute déjà que nous parlons des numéros Montréal Musiques Actuelles ${ }^{1}$ et Musique actuelle?2. C'est un euphémisme de dire qu'avec ces numéros, le cofondateur (en tandem avec Lorraine Vaillancourt) et premier rédacteur en chef de Circuit, Jean-Jacques Nattiez, ne s'était pas fait que des amis! D'ailleurs, à l'occasion d'un numéro signalant, il y a dix ans, les vingt ans de la revue, Nattiez avait signé un article intitulé «La fondation de Circuit et sa première décennie : un coup d'œil rétrospectif et autocritique ${ }^{3}$ ", qui constitue un récit de ce qu'il avait vécu à travers ces numéros et de ce qu'il ferait différemment (avec plus de nuances, notamment, admet-il avec du recul). Rappelons que le numéro Montréal Musiques Actuelles relate la tenue à Montréal de l'édition de 1990 du festival New Music America (aussi nommé, pour cette édition, Montréal Musiques Actuelles), sous la direction artistique de Jean Piché avec qui le débat situé au cœur de la publication se joue (pour faire court: à quel point doit-on être inclusif ou exclusif esthétiquement?). Le numéro Musique actuelle?, pour sa part, publié en 1995 - donc il y a exactement 25 ans cette année -, proposait notamment un dossier tentant de « définir la
1. Vol. 1, $\mathrm{n}^{0} 2,1991$.

2. Vol. $6, n^{\circ} 2,1995$. Notons ici que la troisième polémique évoquée concernait plutôt le débat de l'accessibilité de la musique contemporaine, dans le numéro Ruptures? (vol. 7, $\left.\mathrm{n}^{0} 1,1996\right)$.

3. Vol. $20, n^{\text {os }} 1-2$, p. $13-23$. 
4. Voir: www.supermusique.qc.ca/fr/ accueil (consulté le 14 mars 2020).

5. On peut certes penser ici au travail sur la "comprovisation" de Sandeep Bhagwati, compositeur dont l'arrivée à Montréal, en 2006, plus particulièrement à l'Université Concordia, a eu un impact tangible sur les approches hybridant composition et improvisation. À ce sujet, nous renvoyons nos lecteurs à Jin-Ah Kim (2018), "Réflexions sur la comprovisation de Sandeep Bhagwati : théorie et pratique", Circuit, musiques contemporaines, vol. $28, n^{\circ} 1$, p. 23-33. musique actuelle», avec la participation des principaux musiciens gravitant autour de Productions SuperMusique ${ }^{4}$, un organisme qui fête cette année ses 40 ans (dans ce numéro ayant fait des vagues, les étiquettes collées à l'écriture et à l'improvisation étaient, pour le moins, contrastées et les positions, bien campées). Où en sommes-nous aujourd'hui, en 2020? Sans aucune envie de ressasser le passé et de déterrer de vieilles haches de guerre, nous avons souhaité réinterroger ce phare de la scène montréalaise des musiques improvisées qu'est Productions SuperMusique, tout en nous intéressant à des pratiques impliquant l'improvisation ailleurs qu'à Montréal (particulièrement en France et aux États-Unis), ceci sous un angle bien spécifique. En effet, ce numéro propose de sonder d'une oreille nouvelle la question du continuum improvisation/composition', à partir d'une série d'études de cas, d'entretiens et de témoignages permettant d'examiner précisément différents points de ce continuum (et d'explorer ainsi la subtilité et la complexité des nuances interstitielles, plutôt que de s'en tenir au binarisme de deux postures opposées).

S'il est largement admis aujourd'hui que toute improvisation implique une dialectique du prédéterminé et de l'indéterminé, il reste encore à mieux comprendre les modalités mêmes de ce battement. Par exemple :

- Quel est le statut accordé par les musiciens à ces éléments prédéterminés? Simples conditions de la performance? Ressources individuelles? Facilitateurs de la coordination entre les musiciens? Obstacles, limitations, contraintes?

- Comment ces éléments sont-ils fixés? Par quels types de processus? Quelles formes prennent les opérations de prédétermination? Matérielles, symboliques, cognitives? De quelles manières (et dans quelles mesures) ces opérations viennent-elles réguler le cours de la performance?

- A contrario, quelles stratégies les musiciens utilisent-ils pour générer de l'indétermination ou pour contourner (voire pour détourner) les cadres, codes et conventions qui sous-tendent leurs performances?

Ce dossier permettra peut-être d'esquisser un premier ensemble de réponses à ces différentes questions, tout en soulignant, en filigrane, les 40 ans de Productions SuperMusique. Il est construit en quatre pans: 1) trois articles de fond, 2) divers entretiens et témoignages, 3) un Cahier d'analyse et 4) une rubrique Actualités.

En ouverture du numéro, Clément Canonne signe un article rapportant les résultats principaux d'une enquête ethnographique effectuée en 2016 
sur le processus de création du projet Sillon - une collaboration entre le compositeur Karl Naëgelen et un ensemble d'improvisateurs (Pierre-Antoine Badaroux, Sébastien Beliah, Antonin Gerbal, Ėve Risser et Joris Rühl). Il propose d'éclairer ainsi les spécificités et singularités que ces musiciens attachent à leurs différentes pratiques, par-delà leur volonté commune de parvenir à produire un objet musical hybridant composition et improvisation. Ensuite, Emily Payne et le pianiste Philip Thomas nous offrent un parcours dans l'œuvre de Christian Wolff, depuis Prose Collection (1968) jusqu'aux pièces plus récentes composées pour l'ensemble Apartment House - l'ensemble de Thomas. Cet article examine toutes les «trouvailles» notationnelles utilisées par Wolff pour conférer aux performances de ses pièces ce sens du jeu, de l'immédiateté et de l'interaction si caractéristique de l'improvisation collective. Ce premier pan se termine avec un article de l'organiste Thomas Lacôte, qui est titulaire de l'orgue de l'église de la Sainte-Trinité, ce qui en fait à cet égard un successeur d'Olivier Messiaen. Son article aborde les enjeux propres à sa double activité d'improvisateur et de compositeur, toujours en prise avec les particularités de l'orgue de la Sainte-Trinité, et met en évidence la cardinalité du lieu et de l'instrument dans un processus de création qui ne peut se faire «qu'en présence du son ». Cette contribution permet en outre d'introduire un certain ancrage historique essentiel au dossier, une tradition très singulière de l'improvisation étant associée à l'orgue.

Une série de textes plus brefs constitue le second pan du numéro, en donnant la parole à des musiciens «tout terrain ", aussi à l'aise avec l'improvisation qu'avec la composition ou l'interprétation. Jean-Luc Guionnet ${ }^{6}$ utilise la forme dialoguée - traces des discussions qu'il a pu avoir au gré de rencontres musicales - pour éclairer d'un jour nouveau la relation de l'improvisation à la composition à partir de la distinction entre science-fiction et utopie. Guionnet suggère que l'improvisation s'apparente fondamentalement à une forme de science-fiction, qui ne cherche pas tant à réaliser un préalable idéal faisant fi des circonstances - d'où les «excuses» invoquées en chaîne par les interprètes devant l'impossibilité manifeste de la tâche - qu'à anticiper toutes les conséquences de son inscription dans un temps et un espace donné. Pierre-Antoine Badaroux et Sébastien Beliah rendent compte, quant à eux, de la collaboration entre l'Ensemble Hodos (qu'ils ont fondé en 2008) et le compositeur Philip Corner? C'est l'occasion pour les deux musiciens de revenir sur les questions soulevées par l'interprétation d'œuvres «indéterminées» et de partager, à travers quelques exemples, les réponses singulières que peuvent leur apporter des musiciens improvisateurs. Enfin, dans un entretien avec Clément Canonne, Vincent Lê Quang (saxophoniste et
6. Coauteur, avec Bertrand Denzler, d'un ouvrage récent méritant d'être signalé ici: The Practice of Musical Improvisation: Dialogues with Contemporary Musical Improvisers (Londres, Bloomsbury, 2020).

7. Ce travail a notamment débouché sur une série de trois enregistrements sous étiquette Umlaut (www. umlautrecords.com/u/category/records [consulté le 14 mars 2020]). 
8. Il convient ici de remercier Cléo Palacio-Quintin de son implication, elle qui est à la fois une très proche collaboratrice de SuperMusique et membre de notre comité de rédaction.

9. Ana Dall'Ara-Majek (2018), "Le trésor de la langue de René Lussier: documentation d'un voyage, de la quête linguistique au débat politique", Circuit, musiques contemporaines, vol. $28, n^{\circ} 3$, p. 71-86.

10. Festival international de musique actuelle de Victoriaville (voir: www.fimav.qc.ca/fr [consulté le 14 mars 2020]).

11. Karine P. Bouchard (2020), "L'écoute du Telharmonium 2.0 de Xavier Ménard et Camille St-Amand: les technologies électromécaniques au sein de l'espace d'exposition", Circuit, musiques contemporaines, vol. $30, n^{0} 1$, p. $65-76$.

12. Rappelons que le même Legendre avait aussi publié ici, en 2013, un compte rendu d'un ouvrage précédant de Beaucage portant, celui-là, sur la SMCQ : Éric Legendre (2013), "La Société de musique contemporaine du Québec: histoire à suivre de Réjean Beaucage", Circuit, musiques contemporaines, professeur d'improvisation au Conservatoire de Paris) se livre à une fascinante exploration du soundpainting. Déminant les critiques caricaturales dont cette pratique peut parfois faire l'objet, Lê Quang témoigne à la fois de la puissance du langage - capable de créer les textures les plus complexes à partir de signes très simples - et de son ouverture - dans les espaces d'indétermination qu'il crée sans cesse, pour le soundpainter comme pour les performeurs.

À cela s'ajoute la rubrique Enquête, elle aussi constituée de témoignages d'artistes. Cette collection de textes fait directement référence au dossier «Définir la musique actuelle? ?, paru il y a 25 ans cette année dans le numéro Musique actuelle?. Nous retrouvons ainsi les plumes franches et vivantes de Michel F Côté, Jean Derome, Joane Hétu et Danielle Palardy Roger. Nous les remercions d'avoir - bons joueurs - accepté notre invitation ${ }^{8}$. Un cinquième texte d'un musicien anglophone de la nouvelle génération, Scott Thomson, vient compléter ces témoignages et réflexions qui couvrent les 40 années de créativité de Productions SuperMusique.

Le dossier se poursuit tout naturellement avec, en guise de troisième pan, un Cahier d'analyse signé par Ana Dall'Ara-Majek, qui continue de sonder le «continuum de l'improvisation » en étudiant deux œuvres des directrices (et fondatrices) de Productions SuperMusique: TAGS (2018) de Joane Hétu, et TanGRAM - Orion (2016) de Danielle Palardy Roger. Notons au passage que Dall'Ara-Majek a signé en 2018 un autre Cahier d'analyse pour nos pages, consacré au Trésor de la langue (1989) de René Lussier9 (une œuvre sans partition), tandis que notre plus récent texte pour cette rubrique était destiné à une installation sonore présentée au FIMAV ${ }^{10}$ à l'été 2019: Telharmonium 2.0 de Xavier Ménard et Camille Saint-Amand ${ }^{11}$. C'est dire qu'il est maintenant acquis que, sans renier le moins du monde la partition traditionnelle, les œuvres étudiées dans notre rubrique Cahier d'analyse ne s'y limitent plus.

Enfin, le numéro offre comme quatrième et dernier pan quelques Actualités. D’abord, Éric Legendre propose un compte rendu d'un livre publié par Réjean Beaucage sur la musique actuelle, plus spécifiquement autour du Fimav et de quelques autres festivals ${ }^{12}$. Puis, Clément Canonne et Isaiah Ceccarelli se partagent les Nouveautés en bref en commentant quatre disques. Canonne chronique les enregistrements de François Cotinaud avec l'ensemble Multilatérale et de Sébastien Roux avec l'ensemble Dedalus, qui expérimentent, chacun à leur manière, la relation de l'indéterminé au prédéterminé. Ceccarelli, quant à lui, se concentre sur deux parutions récentes diffusées par DAME (Distributions Ambiances Magnétiques Etcetera), maison de disque créée par Joane Hétu en 1991: l'une de Jean Derome, l'autre de Gabriel Dharmoo. 
Le tout est élégamment illustré d'œuvres de l'artiste Sarah Bahr, qui a réalisé spécialement pour ce numéro «une série de monotypes, un procédé d'impression sans gravure qui produit un tirage unique. S'y jouent l'évocation d'un geste, la tentative de communiquer une vitesse, une articulation, un timbre, un parcours [...] [u]n tracé entre improvisation et composition ${ }^{13}$.»

En terminant, c'est avec un mélange de tristesse et de gratitude que l'équipe de Circuit tient à rendre chaleureusement hommage à la musicologue Flavia Gervasi qui, après une longue et courageuse lutte contre la maladie, est décédée en Italie, bien trop jeune, le 11 février 2020. Flavia a signé deux contributions pour la revue - une enquête autour de Glenn Gould ainsi qu’un entretien avec José Evangelista ${ }^{14}$ - et fut membre de comité de rédaction de 2016 à 2019 .

Merci, Flavia...

Bonne lecture!

Montréal et Paris, mars 2020

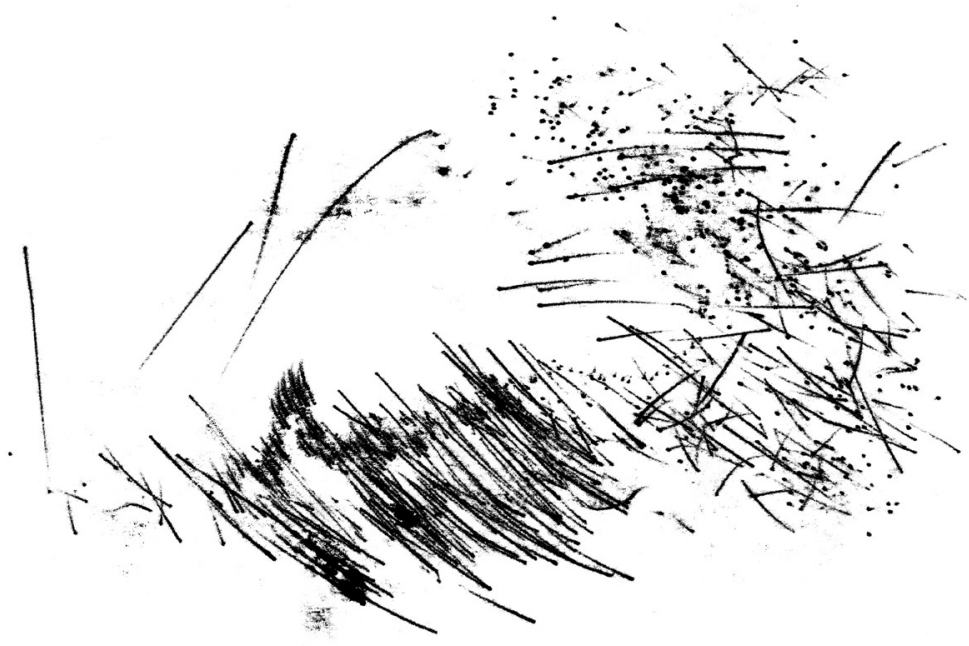

Sarah Bahr, Circles ${ }^{\circ} 3 / 9$ : time is digital, 2020. Encre d'imprimerie sur papier, $42 \times 33 \mathrm{~cm}$.
13. Voir le texte "Les illustrations" à la fin de ce numéro.

14. On trouvera ici le détail de ses collaborations avec Circuit: https://revuecircuit.ca/auteurs/ gervasi_fl (consulté le 14 mars 2020). 